\title{
Creation of gene pools with amplified fragment length polymorphis markers for development of winter oilseed rape (Brassica napus L.) hybrid cultivars
}

\author{
Alina Liersch (D) Jan Bocianowski ( D - Wiesława Popławska • Laurencja Szała • \\ Katarzyna Sosnowska $\cdot$ Teresa Cegielska-Taras $\cdot$ Kamila Nowosad ( \\ Marcin Matuszczak • Iwona Bartkowiak-Broda
}

Received: 9 November 2018/Accepted: 14 January 2019/Published online: 18 January 2019

(C) The Author(s) 2019

\begin{abstract}
The genetic diversity of two winter oilseed rape (Brassica napus L.) collections was investigated using amplified fragment length polymorphism marker technology. The first collection of 78 genotypes was analyzed using 11 primer-enzyme combinations and PCR products were resolved by $13.35 \%$ denaturing polyacrylamide gels. The second collection was investigated using seven fluorescent-labeled AFLP primer combinations and PCR products were separated using capillary electrophoresis. A total of 262 polymorphic AFLP markers were obtained for the first collection and 423 polymorphic markers for the second. On the basis of all markers, principal component analysis was performed for both collections separately. The unweighted pair group method with
\end{abstract}

A. Liersch · W. Popławska · L. Szała ·

K. Sosnowska · T. Cegielska-Taras .

M. Matuszczak · I. Bartkowiak-Broda

Department of Oilseed Crops, Plant Breeding and

Acclimatization Institute - National Research Institute,

Strzeszyńska 36, 60-479 Poznan, Poland

\section{J. Bocianowski ( $\bowtie)$}

Department of Mathematical and Statistical Methods,

Poznań University of Life Sciences, Wojska Polskiego 28, 60-637 Poznan, Poland

e-mail: jan.bocianowski@up.poznan.pl

K. Nowosad

Department of Genetics, Plant Breeding and Seed Production, Wrocław University of Environmental and Life Sciences, Grunwaldzki 24A, 53-363 Wrocław, Poland arithmetic method based on the coefficient of dissimilarity separated the genotypes in collection 1 into two clusters and those in the second collection into three distinct clusters. Resynthesized lines formed a cluster that was clearly distinct from 42 winter oilseed rape parental lines in the cytoplasmic male sterility ogura system. Analysis of molecular variance showed that $79 \%$ and $81 \%$ of the detected variation was found to be within the groups (in the two collections, respectively), while the variation between groups contributed, respectively, to only $21 \%$ and $19 \%$ of the variance. Our results indicate that AFLP technology can be useful for the creation of a gene pool of parental components of winter oilseed rape hybrid cultivars. Additionally, de novo resynthesized Brassica napus lines provide a significant opportunity for enrichment of the gene pool of winter rapeseed.

Keywords winter oilseed rape (Brassica napus L.) · AFLP markers - Genetic diversity · Gene pools · Hybrid breeding

\section{Introduction}

Winter oilseed rape (WOSR, rapeseed, canola) (Brassica napus L. var. oleifera) is the result of spontaneous, interspecific hybridizations between genotypes of turnip rape (Brassica rapa L. syn. campestris; genome AA, $2 n=20$ ) and cabbage (Brassica 
oleracea L.; genome CC, $2 n=18)$. Genetically, the rapeseed is an allopolyploid (genome AACC, $2 n=38$ ) and contains full genomes of turnip rape and cabbage. From being a marginal species grown on a limited area in the mid-twentieth century, rapeseed has become the most important oil crop in countries with a moderate climate, including Poland. Next to soybean, it is the main global oil crop. The significant increase in interest in this plant is the result of intensive breeding, which has resulted in the elimination of erucic acid (C22:1), which is undesirable in human nutrition, from oil composition, and the nearly tenfold reduction in the content of sulfur compounds-glucosinolates in the seeds, which limit the use of post-extraction rapeseed meal as feed for livestock. Double low '00' varieties, i.e., low-erucic, low-glucosinolates ones, have been commonly found in cultivation since the late 1980s. The most important achievements in rapeseed breeding include an improvement of yielding ability and, above all, the development and introduction into cultivation of hybrid varieties (Wittkop et al. 2009). The increase in rapeseed production need not only be a consequence of increased cultivation area, which is limited, but might primarily be the result of the implementation of new technologies of cultivation and biological progress in breeding techniques. The use of molecular/genetic markers enables the selection of economically important features at an early stage of plant development, giving the breeder an additional tool to increase the efficiency of the breeding process (Mikołajczyk 2013; Rakoczy-Trojanowska et al. 2017a, b).

Rapeseed is a relatively young species, which, as a cultivated plant, appeared in Europe only 500 years ago. Currently, it is primarily the natural variability observed within the species that is used. This is relatively narrow due to the limited range of cultivation, absence of wild progenitors acting as a natural source of variation to be used to increase genetic diversity, and the intensive breeding aimed at obtaining double low forms (low-erucic, low-glucosinolates) (Delourme et al. 2013). For this reason, the selection of parental components for combination is a particularly important issue. Such components should be as genetically diverse as possible. Numerous research centers have created the concept of the elaboration, for the purposes of cultivation, of separate genetic pools on the basis of the DNA polymorphism of breeding material. The process of breeding hybrid varieties requires a large number of crosspollination combinations of parental lines and their evaluation in multiple field experiments, preferably in multiple environments in terms of general and specific combining ability, with simultaneous very careful selection of the components based on a number of qualitative characteristics (Bocianowski et al. 2016). For this purpose, methods are sought that would allow the early selection of parental components of F1 hybrids, and thus the shortening of the breeding cycle (Liersch et al. 2016). Such studies performed in different species of plants have shown that one of the elements allowing a high heterosis effect to be obtained in the yield of seeds is the genetic distance (GD) of the parental forms used to make the F1 hybrid. According to the theory of heterosis, the effect of heterosis is greater in hybrids when parental lines are more distant genetically (Fu et al. 2014). A degree of GD between parents is necessary for significant heterosis but it is not sufficient to guarantee it. The relationship between GD and the heterosis effect has been demonstrated, among others, in cereals, corn and rapeseed (Reif et al. 2003; Krystkowiak et al. 2009; Girke et al. 2012a). These results indicate the possibility of the selection of parental lines of hybrids based on their polymorphism identified through observation of phenotypic traits and genotype analysis (Lefort-Buson et al. 1988; Yu et al. 2005). These studies initially involved phenotypic characterization (Ali et al. 1995), isoenzymatic markers (Chèvre et al. 1991), and the development of molecular genetics. Then, methods of genotyping such as AFLP, SSR, SNP and DArTseq led to the possibility of the quick evaluation of genetic variability independent of modifying environmental effects (Stępień et al. 2007; Irzykowska and Bocianowski 2008; Krzymińska et al. 2008; Li et al. 2011; Gao et al. 2014; Wu et al. 2014; Seidler-Łożykowska et al. 2014; Varghese et al. 2014; Rahman et al. 2015; Luo et al. 2016).

The objective of this study was to use AFLP markers to determine the extent of DNA polymorphism to enable estimation of the GD between parental lines of CMS ogura F1 hybrids, double lines and cultivars, as well as resynthesized (RS) B. napus lines and double low-quality semi-RS lines with the restorer gene $(R f o)$. 


\section{Materials and methods}

Plant materials

The plant materials used in this study consisted of two collections of WOSR genotypes from the collection of Plant Breeding Company Strzelce Ltd., Poland, and resynthesized (RS) lines from the Plant Breeding and Acclimatization Institute-NRI. The former included 78 genotypes: 40 CMS ogura male sterile inbred lines, 26 restorer lines for ogura system (Rfo) and 12 cultivars and genotypes of Polish, Ukrainian and Asian origin. The second collection comprised nine CMS ogura male sterile lines, 33 restorer lines $(R f o)$, five RS WOSR lines, and a double low semi-RS line with the Rfo gene.

DNA isolation and molecular marker analysis

Genomic DNA was extracted from young leaves of ten plants from each line using a modified cetyltrimethylammonium bromide (CTAB) extraction protocol (Doyle and Doyle 1990). DNA quantity and purity were checked spectrophotometrically at $260 \mathrm{~nm}$ and $280 \mathrm{~nm}$ wave lengths (NanoDrop 2000, Thermo Scientific) and by $0.8 \%$ agarose gel electrophoresis for $1 \mathrm{~h}$ at $100 \mathrm{~V}$ in $1 \times \mathrm{TBE}$, visualized under UV light after staining with Roti $^{\circledR}$-Safe GelStain (Carl Roth, Karlsruhe, DE). AFLP analysis followed standard methods described by Vos et al. (1995) and in accordance with the manufacturer's instructions: Gibco BRL, AFLP Analysis Reagent Kit, AFLP Analysis System I for collection 1, while collection 2-AFLP ${ }^{\circledR}$ Plant Mapping Kit, Applied Biosystems, Foster City, CA. The primer combinations were selected from the linkage map for rapeseed described by Lombard and Delourme (2001) and the results in the analyses of collections of different WOSR genotypes found in Liersch et al. (2013).

Genomic DNA of both collections of oilseed rape were digested with the restriction enzymes EcoRI and MseI (AFLP Core Reagent Kit, Invitrogen). Eleven primer combinations (EcoRI, MseI) (Gibco BRL, AFLP Starter Primer Kit) were used in selective amplifications of WOSR collection 1. PCR products were resolved by $13.35 \%$ denaturing polyacrylamide gels, and the gels were stained with silver. Seven primer combinations were used in selective amplifications of WOSR collection 2. EcoRI primers were fluorescently labeled with blue (FAM), green (JOE) and yellow (NED) 5' reporter dyes (AFLP Selective Amplification Primers, Applied Biosystems). Amplification products were separated using capillary electrophoresis on an ABI Prism 3130xl Genetic Analyzer (Applied Biosystems) and analyzed by PeakScanner 1.0 (Applied Biosystems).

Data scoring and statistical analysis

A peak was considered polymorphic if it was present in at least one accession and absent in others. Mostly, clear peaks (markers) with strong intensity were counted, and only clear-cut polymorphic peaks (markers) were scored, and the data were exported to a 1-0 matrix for statistical evaluation. Fragments smaller than 50 bp were excluded from the data matrix. Both collections were analyzed independently. The polymorphic information content (PIC) for each primer was calculated using the formula described by Wolko et al. (2015):

PIC $=1-\sum_{i=1}^{n} p_{i}^{2}$,

where $p_{i}$ is the frequency of the $i$-th allele and $n$ is the number of markers per primer. PIC values ranged from 0 (in the case of fixation of one allele) to 0.5 (when the frequencies of both alleles were equal). The data matrix for both collections was used to calculate pairwise GD based on Nei and Li (1979) coefficients. Genetic distance for each pair of genotypes was estimated based on the coefficient proposed by Nei and $\mathrm{Li}$ (1979), defined as $\mathrm{GS}=2 \mathrm{~N}_{\mathrm{XY}} /\left(\mathrm{N}_{\mathrm{X}}+\mathrm{N}_{\mathrm{Y}}\right)$, where $N_{X Y}$ is the number of bands shared by genotypes $\mathrm{X}$ and $\mathrm{Y}, \mathrm{N}_{\mathrm{X}}$ is the number of bands in genotype $X$, and $N_{Y}$ is the number of bands in genotype $\mathrm{Y}$. The genotypes were grouped using the unweighted pair group method with arithmetic mean (UPGMA). Dissimilarities between genotypes were visualized on a dendrogram. On the basis of all markers, principal component analysis (PCA) was performed with the use of the GenStat v. 18 statistical package. This multivariate technique allowed us to find and plot the major pattern within the multiple loci data set. The resulting distance matrix was also subjected to an analysis of molecular variance (AMOVA) using GenAlEx 6.5 (Peakall and Smouse 2012). AMOVA estimated and partitioned the total 
molecular variance within and between groups of genotypes and then tested the significance of partitioned variance components using nonparametric permutational testing procedures with 999 permutations (Excoffier et al. 1992). The $F_{S T}$ coefficient was used as a measure of population genetic structure and was calculated from the formula: $F_{S T}=\left(H_{T}-H_{S}\right) /$ $H_{T}$, where $H_{T}$ is the probability that two alleles drawn at random (with replacement) from the entire population differ in state (i.e., the probability of no population structure), and $H_{S}$ is the probability that two alleles drawn at random from a subpopulation differ in state (which, for a two-allele system, will always be $2 p_{i} q_{i}$, where $p_{i}$ is the observed allelic frequency in subpopulation $i$ ), averaged over subpopulations. Groups for AMOVA were identified on the basis of the pedigree of the genotypes (Table 1).

\section{Results}

The first collection of 78 genotypes of WOSR was tested using 11 combinations of AFLP primers. 539 DNA amplification products were obtained, of which 262 were polymorphic (Table 2). The number of polymorphic markers for a single primer combination ranged from 14 to 36 . In the reaction with the EAGG: MCTA primers the highest number of polymorphic markers was obtained-36, while the highest level of polymorphism was found with the primer combinations EAGG: MCTA-59.00\% and EAGG: MCTC$71.43 \%$. One primer combination generated an average of 23.8 polymorphic DNA fragments. The set of AFLP primers used in this study generated highly informative loci with PIC values ranging from 0.474 to 0.500 , with a mean of 0.491 (Table 2). The GD data matrix based on the Nei and Li (1979) coefficients ranged from 0.08 to 0.74 and the lowest was for genotype pairs DG 25 and DG 26 (0.08) and DG 7 and DG 8 and DG 73 and DG 78 (0.09) and the highest for genotypes DG 9 and DG 52 (0.74) as well as DG 2 and DG 55-0.72 (Table 3). The dendrogram obtained by cluster analysis of the Nei and Li (1979) distance values identified 78 genotypes in two basic groups-A and $\mathrm{B}$ - each of which was divided into smaller clusters (Fig. 1). The first group (A) is partitioned into three smaller subgroups. In the first (A1) are three cultivars from Ukraine: 'Szmaragd', 'Królowa Śniegu' and 'Kleopatra' (DG 1-DG 3), 24 CMS ogura lines (DG 4-DG 27), and GMS line DG 28. The second small group (A2) contained two GMS lines (DG 29, DG 30), and nine CMS ogura lines (DG 31DG 39). The third (A3) small cluster comprised only three CMS ogura lines (DG 40-DG 42). The second group (B) is also divided into three subgroups. The first subgroup (B1) is the most diverse, because it includes both "00" lines of WOSR (DG 44-DG 46), four lines with the restorer gene Rfo (DG 53-DG 56), the Chinese line resistant to Sclerotinia sclerotiorum (DG 52) and three CMS ogura lines (DG49-DG 51). The second small cluster (B2) contained only yellow seeded restorer lines $R f o-$ numbers from DG 69 to DG 78. The last (B3) small cluster included only 12 lines with the restorer gene $R f o-$ numbers from DG 69 to DG 78 (Fig. 1).

AMOVA of WOSR of different genotypes (cultivars, double low, CMS and restorer lines for Ogura system, Table 1) as a grouping criterion (four groups) revealed that within-group variance accounted for $79 \%$ of the total variance, while variation between groups contributed $21 \%$ (Table 4 ). The significant differentiation $\left(F_{S T}=0.215 ; p=0.001\right)$ between four groups was supported by the results of AMOVA (Tables 4 and 5). PCA was performed to confirm the structure and the diversity of genotypes of WOSR based on all markers (Fig. 2). The first two principal components explained a total of $23.18 \%$ of marker variation (14.71, and $8.47 \%$, respectively). The first axis, which accounted for $14.71 \%$ of the variance, separated the genotypes into two clusters. The second axis accounted for $8.47 \%$ of the variance, and showed the diversity of two groups of genotypes.

AFLP fingerprinting of the 48 genotypes from collection 2 with seven EcoRI/MseI primer combinations detected a high level of polymorphism-423 polymorphic AFLP markers (Table 2). The number of polymorphic markers per primer combinations ranged from 24 (EACT FAM: MCTC) to 86 (EACC NED: MCTC), with an average of 60.4 markers. Primer combinations EAGG JOE: MCTA-93.44\%, EACC NED: MCTC - $93.48 \%$ and EACC NED: MCAG$96.0 \%$ were the most effective in detecting polymorphism among genotypes. The set of AFLP primers used for collection 2 generated highly informative loci with PIC values ranging from 0.423 to 0.500 , with a mean of 0.484 (Table 2). The GD coefficient showed the lowest dissimilarity value: 0.14 for CMS ogura lines (DG 13/14 and DG 14/14), and the highest value 
Table 1 List of plant material (B. napus genotypes and RS lines) with their codes used in the study, group number for analysis of molecular variance, type and country of origin

\begin{tabular}{|c|c|c|c|c|c|}
\hline $\begin{array}{l}\text { Genotype } \\
\text { code/group }\end{array}$ & $\begin{array}{l}\text { Type/country of } \\
\text { origin }\end{array}$ & $\begin{array}{l}\text { Genotype } \\
\text { code/group }\end{array}$ & Type/country of origin & $\begin{array}{l}\text { Genotype } \\
\text { code/group }\end{array}$ & $\begin{array}{l}\text { Type/country of } \\
\text { origin }\end{array}$ \\
\hline \multicolumn{6}{|l|}{ Collection 1} \\
\hline DG $1 / 1$ & Szmaragd, OP, UA & DG $27 / 2$ & CMS ogura, PL & DG 53/4 & $\begin{array}{l}\text { Rfo restorer line, } \\
\text { PL }\end{array}$ \\
\hline DG $2 / 1$ & Kleopatra, OP, UA & DG $28 / 2$ & GMS HEN, PL & DG $54 / 4$ & $R f o, \mathrm{PL}$ \\
\hline DG 3/1 & $\begin{array}{l}\text { Królowa Śniegu, } \\
\text { OP, UA }\end{array}$ & DG $29 / 2$ & GMS HEN, PL & DG 55/4 & $R f o, \mathrm{PL}$ \\
\hline DG $4 / 2$ & CMS ogura, PL & DG 30/2 & GMS HEN, PL & DG 56/4 & $R f o, \mathrm{PL}$ \\
\hline DG $5 / 2$ & CMS ogura, PL & DG $31 / 2$ & CMS ogura, PL & DG 57/4 & $R f o, \mathrm{PL}$ \\
\hline DG $6 / 2$ & CMS ogura, PL & DG $32 / 2$ & CMS ogura, PL & DG 58/4 & $R f o, \mathrm{PL}$ \\
\hline DG $7 / 2$ & CMS ogura, PL & DG $33 / 2$ & CMS ogura, PL & DG 59/4 & $R f o, \mathrm{PL}$ \\
\hline DG $8 / 2$ & CMS ogura, PL & DG $34 / 2$ & CMS ogura, PL & DG 60/4 & $R f o, \mathrm{PL}$ \\
\hline DG $9 / 2$ & CMS ogura, PL & DG $35 / 2$ & CMS ogura, PL & DG $61 / 4$ & $R f o, \mathrm{PL}$ \\
\hline DG $10 / 2$ & CMS ogura, PL & DG $36 / 2$ & CMS ogura, PL & DG $62 / 4$ & $R f o, \mathrm{PL}$ \\
\hline DG $11 / 2$ & CMS ogura, PL & DG $37 / 2$ & CMS ogura, PL & DG 63/4 & $R f o, \mathrm{PL}$ \\
\hline DG $12 / 2$ & CMS ogura, PL & DG $38 / 2$ & CMS ogura, PL & DG 64/4 & $R f o, \mathrm{PL}$ \\
\hline DG $13 / 2$ & CMS ogura, PL & DG $39 / 2$ & CMS ogura, $\mathrm{PL}$ & DG 65/4 & $R f o, \mathrm{PL}$ \\
\hline DG $14 / 2$ & CMS ogura, PL & DG $40 / 2$ & CMS ogura, PL & DG $66 / 4$ & $R f o, \mathrm{PL}$ \\
\hline DG $15 / 2$ & CMS ogura, PL & DG $41 / 2$ & CMS ogura, PL & DG 67/4 & $R f o, \mathrm{PL}$ \\
\hline DG $16 / 2$ & CMS ogura, PL & DG $42 / 2$ & CMS ogura, PL & DG $68 / 4$ & $R f o$, PL \\
\hline DG $17 / 2$ & CMS ogura, PL & DG $43 / 2$ & CMS ogura, PL & DG 69/4 & Rfo, PL, (ys) \\
\hline DG $18 / 2$ & CMS ogura, PL & DG $44 / 3$ & 00 lines, $\mathrm{PL}$ & DG 70/4 & Rfo, PL, (ys) \\
\hline DG 19/2 & CMS ogura, PL & DG $45 / 3$ & 00 lines, $\mathrm{PL}$ & DG 71/4 & Rfo, PL, (ys) \\
\hline DG $20 / 2$ & CMS ogura, PL & DG $46 / 3$ & 00 lines, PL & DG $72 / 4$ & Rfo, PL, (ys) \\
\hline DG $21 / 2$ & CMS ogura, PL & DG $47 / 3$ & 00 lines, $\mathrm{PL}$ & DG 73/4 & $R f o, \mathrm{PL},(\mathrm{ys})$ \\
\hline DG $22 / 2$ & CMS ogura, PL & DG 48/3 & 00 lines, PL & DG 74/4 & Rfo, PL, (ys) \\
\hline DG $23 / 2$ & CMS ogura, PL & DG 49/2 & CMS ogura, PL & DG 75/4 & $R f o, \mathrm{PL},(\mathrm{ys})$ \\
\hline DG $24 / 2$ & CMS ogura, PL & DG 50/2 & CMS ogura, PL & DG 76/4 & Rfo, PL, (ys) \\
\hline DG $25 / 2$ & CMS ogura, PL & DG $51 / 2$ & CMS ogura, PL & DG 77/4 & $R f o, \mathrm{PL},(\mathrm{ys})$ \\
\hline DG $26 / 2$ & CMS ogura, PL & DG $52 / 3$ & $\begin{array}{l}\text { CHO } 11, \mathrm{~s}-\mathrm{w}, \text { resistant to } S . \\
\text { sclerotiorum, CN }\end{array}$ & DG 78/4 & $R f o$, PL, (ys) \\
\hline \multicolumn{6}{|l|}{ Collection 2} \\
\hline DG 1/14/1 & $\begin{array}{l}\text { Rfo restorer line, } \\
\text { PL }\end{array}$ & DG 17/14/1 & Rfo, PL & DG 33/14/1 & $R f_{o}, \mathrm{PL}$ \\
\hline DG 2/14/1 & Rfo, PL & DG 18/14/1 & $R f o, \mathrm{PL}$ & DG 34/14/1 & $R f o, \mathrm{PL}$ \\
\hline DG 3/14/1 & Rfo, PL & DG 19/14/1 & $R f o, \mathrm{PL}$ & DG 35/14/1 & $R f o$, PL \\
\hline DG 4/14/1 & Rfo, PL & DG 20/14/1 & $R f o, \mathrm{PL}$ & DG 36/14/1 & $R f o, \mathrm{PL}$ \\
\hline DG $5 / 14 / 1$ & Rfo, PL & DG 21/14/1 & $R f o, \mathrm{PL}$ & DG 37/14/1 & $R f o, \mathrm{PL}$ \\
\hline DG $6 / 14 / 1$ & Rfo, PL & DG 22/14/1 & $R f o, \mathrm{PL}$ & DG 38/14/1 & $R f o, \mathrm{PL}$ \\
\hline DG $7 / 14 / 1$ & Rfo, PL & DG 23/14/1 & $R f o, \mathrm{PL}$ & DG 39/14/1 & $R f o, \mathrm{PL}$ \\
\hline DG $8 / 14 / 2$ & CMS ogura, PL & DG 24/14/1 & $R f o, \mathrm{PL}$ & DG 40/14/1 & $R f o, \mathrm{PL}$ \\
\hline DG $9 / 14 / 2$ & CMS ogura, PL & DG 25/14/1 & $R f o, \mathrm{PL}$ & DG 41/14/1 & $R f o, \mathrm{PL}$ \\
\hline DG $10 / 14 / 2$ & CMS ogura, PL & DG 26/14/1 & $R f o, \mathrm{PL}$ & RR_10/1 & $R f o, \mathrm{PL}$ \\
\hline DG 11/14/2 & CMS ogura, PL & DG 27/14/1 & $R f o, \mathrm{PL}$ & RS 42/14/3 & $\begin{array}{l}\text { semi-RS line, } \\
\text { Rfo PL }\end{array}$ \\
\hline
\end{tabular}


Table 1 continued

\begin{tabular}{|c|c|c|c|c|c|}
\hline $\begin{array}{l}\text { Genotype } \\
\text { code/group }\end{array}$ & $\begin{array}{l}\text { Type/country of } \\
\text { origin }\end{array}$ & $\begin{array}{l}\text { Genotype } \\
\text { code/group }\end{array}$ & Type/country of origin & $\begin{array}{l}\text { Genotype } \\
\text { code/group }\end{array}$ & $\begin{array}{l}\text { Type/country of } \\
\text { origin }\end{array}$ \\
\hline DG 12/14/2 & CMS ogura, PL & DG 28/14/1 & Rfo, $\mathrm{PL}$ & $\operatorname{RS} 48 / 14 / 3$ & RS line, PL \\
\hline DG 13/14/2 & CMS ogura, PL & DG 29/14/1 & $R f o, \mathrm{PL}$ & RS 50/14/3 & RS line, $\mathrm{PL}$ \\
\hline DG 14/14/2 & CMS ogura, PL & DG 30/14/1 & Rfo, $\mathrm{PL}$ & RS 55/14/3 & RS line, PL \\
\hline DG $15 / 14 / 2$ & CMS ogura, PL & DG 31/14/1 & $R f o$, PL & RS 60/14/3 & RS line, $\mathrm{PL}$ \\
\hline DG 16/14/2 & CMS ogura, PL & DG 32/14/1 & Rfo, PL & RS 67/14/3 & RS line, PL \\
\hline
\end{tabular}

$O P$ open-pollinated, GMS HEN genetic male sterility HEN, $s$ - $w$ semi winter, $y s$ yellow seeded, $U A$ Ukraine, $P L$ Poland, $C N$ China

Table 2 Genetic diversity analysis of investigated WOSR genotypes collection with the use of AFLP primer combinations

\begin{tabular}{|c|c|c|c|c|}
\hline Primer combinations $* *$ & No. of bands & No. of polymorphic bands & Degree of polymorphism (\%) & PIC* \\
\hline \multicolumn{5}{|l|}{ Collection 1} \\
\hline E-ACC: M-CAC & 40 & 14 & 35.00 & 0.488 \\
\hline E-ACC: M-CAG & 44 & 20 & 45.46 & 0.488 \\
\hline E-ACC: M-CTC & 47 & 25 & 53.19 & 0.499 \\
\hline E-ACT: M-CAT & 42 & 14 & 33.33 & 0.474 \\
\hline E-ACT: M-CTC & 45 & 26 & 57.78 & 0.494 \\
\hline E-ACT: M-CTT & 46 & 24 & 52.17 & 0.499 \\
\hline E-AGG: M-CAC & 59 & 22 & 37.29 & 0.494 \\
\hline E-AGG: M-CAG & 48 & 27 & 57.25 & 0.500 \\
\hline E-AGG: M-CAT & 65 & 24 & 36.92 & 0.487 \\
\hline E-AGG: M-CTA & 61 & 36 & 59.00 & 0.481 \\
\hline E-AGG: M-CTC & 42 & 30 & 71.43 & 0.497 \\
\hline Total & 539 & 262 & 48.61 & 0.500 \\
\hline \multicolumn{5}{|l|}{ Collection $2 * * *$} \\
\hline E-AAC NED***: M-CAC & 87 & 80 & 91.95 & 0.486 \\
\hline E-ACC NED***: M-CAC & 65 & 57 & 87.69 & 0.500 \\
\hline E-ACC NED***: M-CAG & 75 & 72 & 96.00 & 0.489 \\
\hline E-ACT FAM***: M-CTC & 27 & 24 & 88.89 & 0.423 \\
\hline E-ACC NED***: M-CTC & 92 & 86 & 93.48 & 0.496 \\
\hline E-AGG JOE***: M-CAC & 54 & 47 & 87.04 & 0.493 \\
\hline E-AGG JOE***: M-CTA & 61 & 57 & 93.44 & 0.498 \\
\hline Total & 461 & 423 & 91.75 & 0.493 \\
\hline
\end{tabular}

* PIC-polymorphism information content

** Primers EcoRI and MseI have three additional bases at the $3^{\prime}$ end

*** Every EcoRI primer a 5' dye-labeled primer: FAM (blue), JOE (green) or NED (yellow)

was 0.74 between restorer line (DG 41/14) and resynthesized oilseed rape line RS48/14 (Table 3). Cluster analysis enabled differentiation of the investigated genotypes into three main groups: C, D, E (Fig. 3). The first group (C) included one CMS ogura line (DG 8/14) and 25 lines with the restorer 
Table 3 Minimum and maximum of genetic distance values calculated according to using Nei and Li (1979) measure

\begin{tabular}{|c|c|c|c|c|c|}
\hline \multicolumn{3}{|c|}{ Minimum genetic dissimilarity value } & \multicolumn{3}{|c|}{ Maximum genetic dissimilarity value } \\
\hline \multicolumn{6}{|l|}{ Collection 1} \\
\hline DG 25 & DG 26 & 0.08 & DG 9 & DG 52 & 0.74 \\
\hline DG 7 & DG 8 & 0.09 & DG 2 & DG 55 & 0.72 \\
\hline DG 73 & DG 78 & 0.09 & DG 10 & DG 52 & 0.69 \\
\hline DG 2 & DG 3 & 0.10 & DG 5 & DG 55 & 0.68 \\
\hline DG 4 & DG 5 & 0.11 & DG 18 & DG 61 & 0.68 \\
\hline DG 73 & DG 77 & 0.12 & DG 38 & DG 52 & 0.68 \\
\hline DG 76 & DG 77 & 0.12 & DG23 & DG 52 & 0.67 \\
\hline DG 74 & DG 75 & 0.12 & DG 23 & DG 54 & 0.67 \\
\hline DG 75 & DG 76 & 0.13 & DG 3 & DG 55 & 0.66 \\
\hline DG 19 & DG 21 & 0.13 & DG 31 & DG 77 & 0.66 \\
\hline \multicolumn{6}{|l|}{ Collection 2} \\
\hline DG 13/14 & DG 14/14 & 0.14 & DG 41/14 & RS 48/14 & 0.74 \\
\hline DG 19/14 & DG 21/14 & 0.17 & DG 5/14 & RS 50/14 & 0.71 \\
\hline DG 7/14 & DG 29/14 & 0.18 & DG 8/14 & RS 50/14 & 0.69 \\
\hline DG 5/14 & DG 31/14 & 0.19 & DG 25/14 & RS50/14 & 0.68 \\
\hline DG 12/14 & DG 13/14 & 0.20 & DG 40/14 & RS 48/14 & 0.68 \\
\hline DG 21/14 & DG 31/14 & 0.20 & DG 18/14 & RS 67/14 & 0.68 \\
\hline DG 37/14 & DG 40/14 & 0.21 & DG $1 / 14$ & RS 48/14 & 0.67 \\
\hline DG 13/14 & DG 19/14 & 0.21 & DG 2/14 & RS 50/14 & 0.67 \\
\hline DG 4/14 & DG $29 / 14$ & 0.21 & DG $1 / 14$ & RS 67/14 & 0.67 \\
\hline DG 28/14 & DG 31/14 & 0.21 & DG 9/14 & RS 60/14 & 0.66 \\
\hline DG 30/14 & DG 40/14 & 0.21 & RR_10 & RS 48/14 & 0.66 \\
\hline
\end{tabular}

gene $(R f o)$. The cluster D comprised 16 genotypes and was subdivided into two smaller clusters. Six CMS ogura lines (DG 11/14-DG 16/14) formed cluster D1, and eight restorer lines (Rfo) and two CMS ogura lines (DG 9/14, DG 10/14) cluster D2. The last group (E) included only resynthesized lines: RS 55/14, RS 60/14, RS 42/14, RS 50/14, RS 48/14, RS 67/14. Analyzing the results of molecular tests of genotypes from collection 2, specific markers appeared only in the resynthesized lines of WOSR. These markers did not occur in the analyzed double low WOSR genotypes, whereas they were present in a few of the resynthesized genotypes. The AFLP markers characteristic for RS genotypes were found in five of the seven AFLP primer combinations used in the study. AFLP markers AAC/CAC $275,276, \mathrm{ACC}_{2} \mathrm{CAC}_{175}$, $\mathrm{ACT} / \mathrm{CTC}_{93}$ and $\mathrm{AGG} / \mathrm{CTA}_{499.8}$ were found in three resynthesized lines, and the $\mathrm{AAC} / \mathrm{CAC}_{306}, \mathrm{ACC} /$ $\mathrm{CAC}_{201}$ and $\mathrm{ACC} / \mathrm{CAG}_{277}$ markers in four RS lines. Seven AFLP markers were found in all analyzed resynthesized lines of WOSR-AAC/CAC 344,416 ,
$\mathrm{ACC} / \mathrm{CAC}_{96,132}, \quad \mathrm{ACC} / \mathrm{CAG}_{278}$ and $\mathrm{AGG} /$ $\mathrm{CTA}_{83.3}, 276.2$, and six AFLP markers in five RS

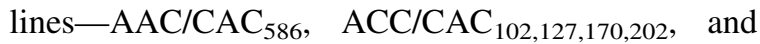
$\mathrm{ACC} / \mathrm{CAG}_{333}$, respectively. AMOVA for the WOSR genotypes, using types (CMS or restorer lines for Ogura system, RS lines) as a grouping criterion (three groups), revealed that within-group variance accounted for $81 \%$ of the total variance, while variation between groups contributed $19 \%$ (Table 4). The significant differentiation $\quad\left(F_{S T}=0.185\right.$; $p=0.001$ ) between the three groups was supported by the results of AMOVA (Tables 4 and 5). Variations in individual groups were similar: 49.4 for CMS ogura lines, 54.2 for resynthesized lines and 55.5 for restorer lines (Table 5). PCA was performed to confirm the structure and to gain insight into the diversity of 48 genotypes from collection 2 based on the 423 AFLP markers (Fig. 4). The first two PCs explained a total of $18.79 \%$ of AFLP marker variation (11.79 and $7.00 \%$, respectively). The first axis, which accounted for $11.79 \%$ of the variance, separated the genotypes 
Fig. 1 Genetic dissimilarity dendrogram of 78 genotypes (collection 1) of WOSR, based on 262 AFLP markers

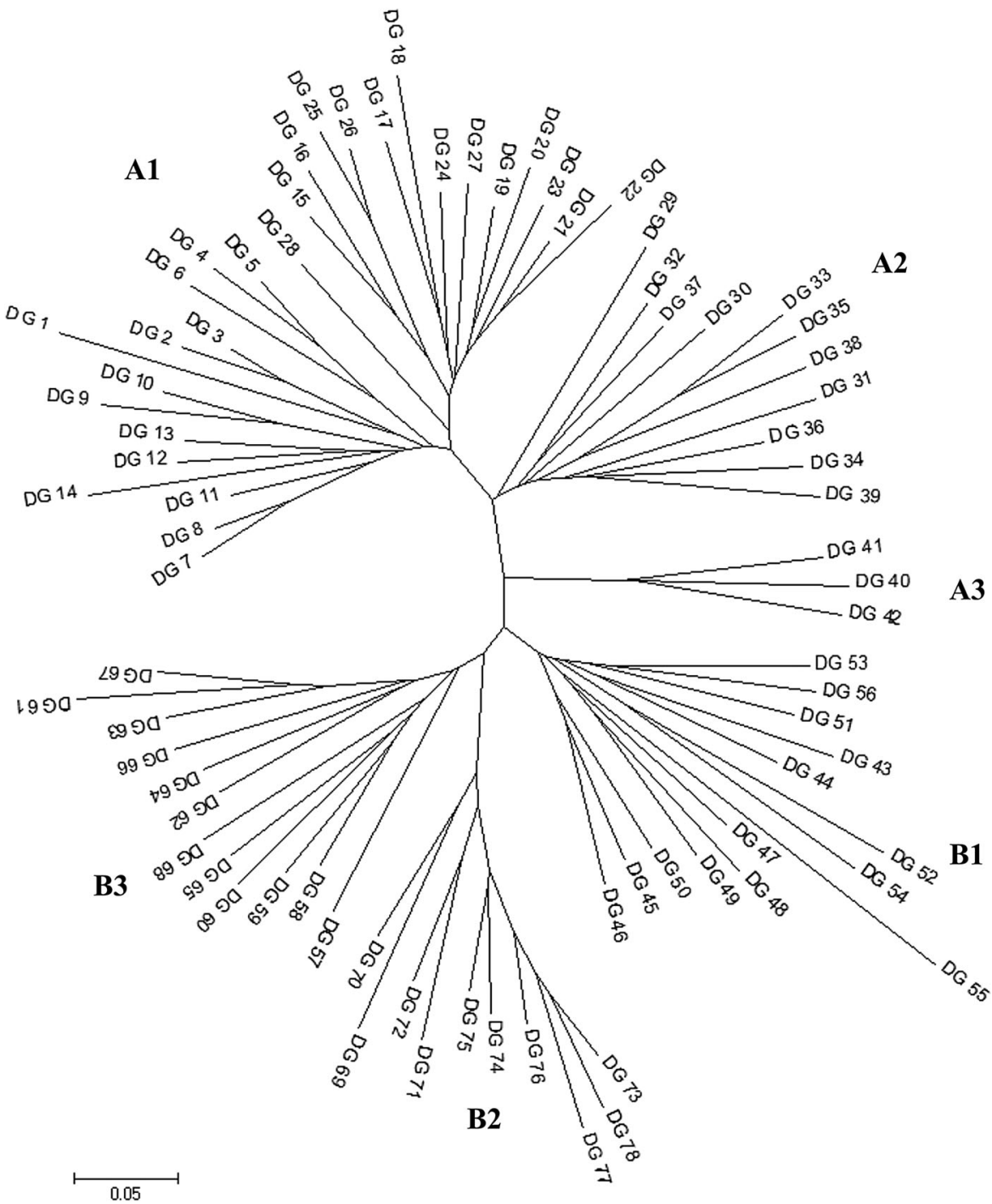

Table 4 Results of analysis of molecular variance (AMOVA) for both genotype collections

\begin{tabular}{|c|c|c|c|c|c|}
\hline Source of variation & Degrees of freedom & Sum of squares & Mean squares & Estimated variance & Percentage of variation (\%) \\
\hline \multicolumn{6}{|l|}{ Collection 1} \\
\hline Among groups & 3 & 606.921 & 202.307 & 10.834 & 21 \\
\hline Within groups & 74 & 2931.374 & 39.613 & 39.613 & 79 \\
\hline Total & 77 & 3538.295 & & 50.447 & 100 \\
\hline \multicolumn{6}{|l|}{ Collection 2} \\
\hline Among groups & 2 & 416.285 & 208.143 & 13.141 & 19 \\
\hline Within groups & 45 & 2603.131 & 57.847 & 57.847 & 81 \\
\hline Total & 47 & 3019.417 & & 70.988 & 100 \\
\hline
\end{tabular}


Table 5 Molecular variance for particular groups of both genotype collections

\begin{tabular}{|c|c|c|c|}
\hline Groups & $\begin{array}{l}\text { Number of genotype in the group/ } \\
\mathrm{N}\end{array}$ & $\begin{array}{l}\text { Sum of squares within group/ } \\
\text { SSWP }\end{array}$ & $\begin{array}{l}\text { Mean square within the group/ } \\
\text { MSWP }\end{array}$ \\
\hline \multicolumn{4}{|l|}{ Collection 1} \\
\hline Cultivars & 3 & 60 & 20 \\
\hline $\begin{array}{l}\text { CMS ogura } \\
\text { lines }\end{array}$ & 43 & 1671 & 38.9 \\
\hline 00 lines & 6 & 178 & 29.7 \\
\hline Rfo lines & 26 & 1022 & 39.3 \\
\hline \multicolumn{4}{|l|}{ Collection 2} \\
\hline $\begin{array}{l}\text { Rfo restorer } \\
\text { lines }\end{array}$ & 33 & 1383 & 55.5 \\
\hline $\begin{array}{l}\text { CMS ogura } \\
\text { lines }\end{array}$ & 9 & 445 & 49.4 \\
\hline$R S$ lines & 6 & 325 & 54.2 \\
\hline
\end{tabular}

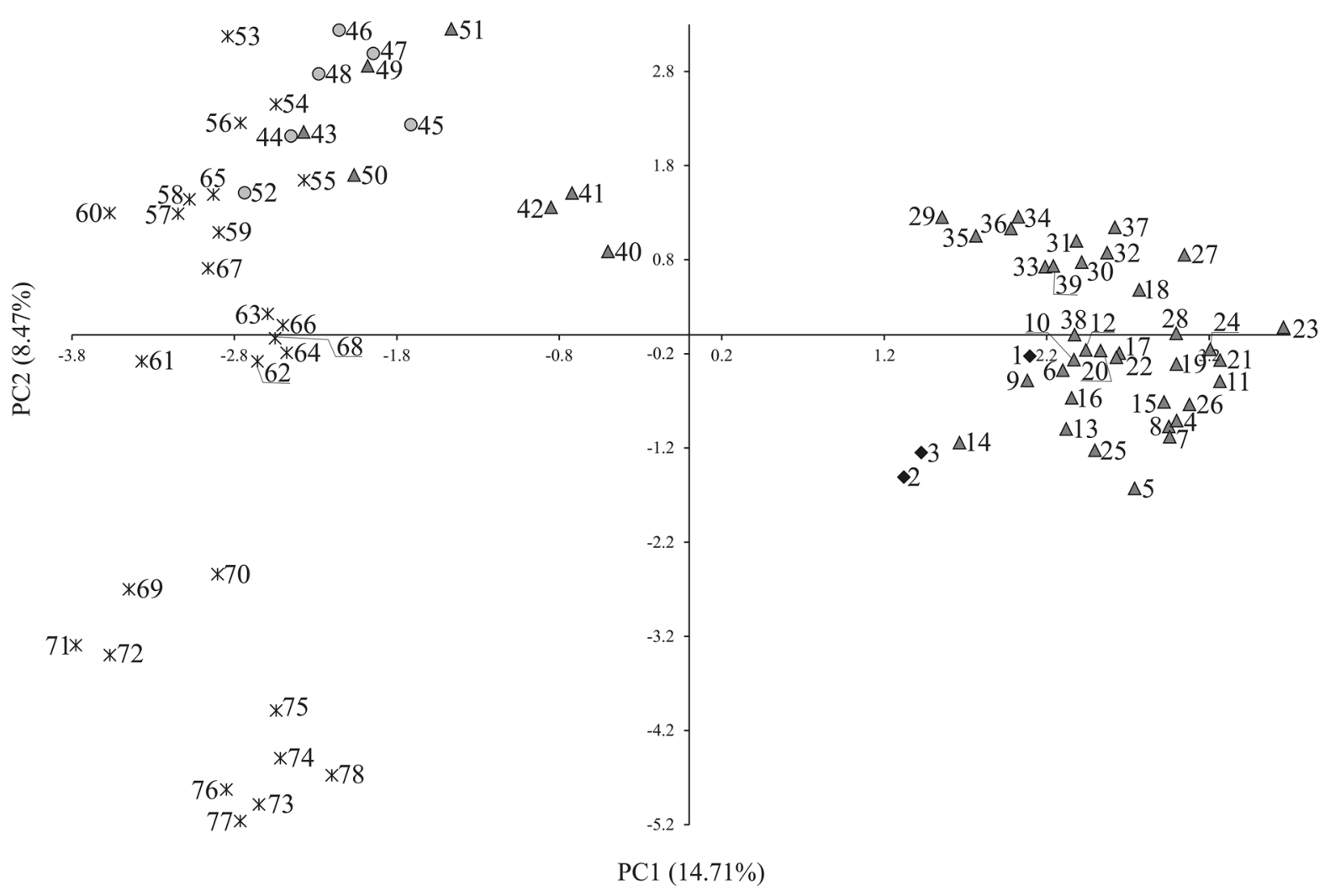

Fig. 2 Principal component analysis (PCA) of 78 genotypes winter oilseed rape (collection 1) based on 262 AFLP markers

which were assigned by UPGMA to the first and second clusters. It is evident that the results provided by these two methods applied independently are complementary.

\section{Discussion}

Research carried out in various species of crops has shown that one of the elements that allows a high 
Fig. 3 Genetic dissimilarity dendrogram of 48 genotypes (collection 2) of WOSR, based on 423 AFLP markers

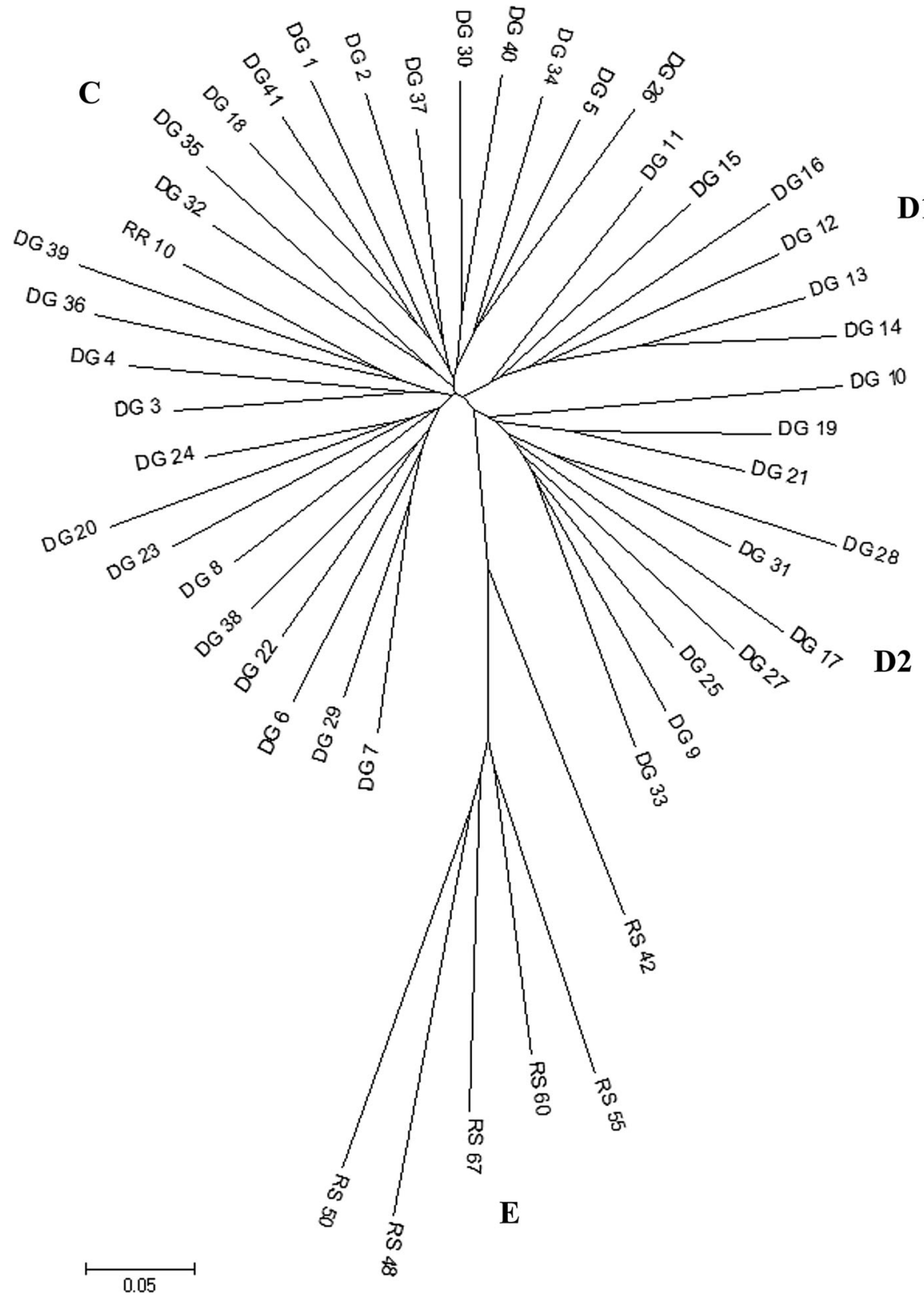

effect of heterosis in the seed yield to be obtained is the GD of parental forms used to create F1 hybrids. Therefore, many breeding companies have formed gene pools based on the DNA polymorphism of breeding materials (Snowdon and Friedt 2004). Molecular analysis of two WOSR genotype collections originating mainly from the Plant Breeding Company Strzelce Ltd. and Plant Breeding and Acclimatization Institute-NRI performed with the use of AFLP molecular markers was to form the basis of the creation of gene pools for the development of the WOSR F1 hybrids. Previous studies on Brassica crops confirmed the usefulness of AFLP markers for analyses of genetic variability (Ecke et al. 2010; Faltusová et al. 2011; Liersch et al. 2013). The choice of the AFLP primer combinations based on restriction enzymes (EcoRI and MseI) was made on the basis of a genetic map of WOSR (Lombard and Delourme 2001) 


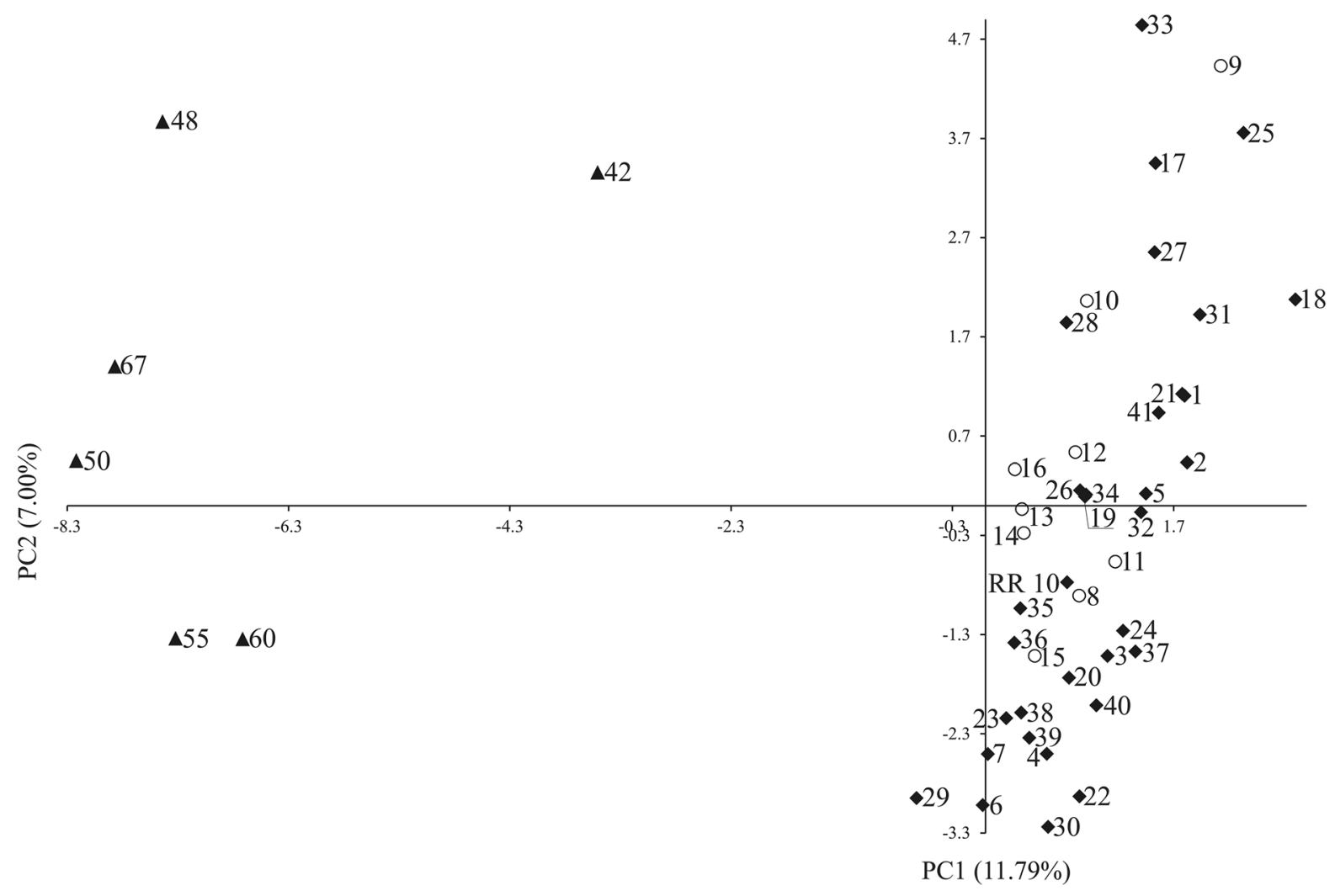

Fig. 4 PCA of 48 genotypes (collection 2) of WOSR, based on 423 AFLP markers

and studies of parental lines of CMS ogura hybrids (Liersch et al. 2013). The results confirmed the usefulness of selected AFLP primer combinations due to the large number of markers obtained for one primer combination-53.9 for the first collection, and 65.9 for the second one, as well as the high efficiency and repeatability of the AFLP method. The analysis of the first collection used the method of separating PCR products on polyacrylamide gel, and detection by silver staining, while in the second study a different method was used for analysis of PCR products using capillary electrophoresis. The use of such a modification made it possible to simplify and significantly accelerate research and reduce the labor intensiveness of the method. Jesske et al. (2013) showed the usefulness of AFLP analysis using fluorescently labeled markers and separation of amplification products on capillaries in studies of genetic diversity of 126 rapeseed genotypes. Nurhasanah and Ecke (2016) indicated that the AFLP method is one of the most efficient methods, generating a large number of DNA markers in the study of genetic variation and mapping.
The authors also demonstrated the usefulness of AFLP and SSR markers for the creation of genetic maps, genomic analyses and quantitative traits loci mapping. 262 AFLP polymorphic markers for the first collection and 423 markers obtained in the studies of the second collection allowed the determination of the genetic diversity within each collection, providing a sufficient assessment of the variability of the examined winter rape genomes. The dendrograms created on the basis of the GD value divided the examined genotypes of both collections into separate groups according to the type of plant material and its origin/pedigree. CMS ogura lines and restorer lines (Rfo) form separate groups in both collections, as do Ukrainian cultivars in the first of the studied collections. Resynthetic lines of winter oilseed rape newly obtained in the Tissue Culture Laboratory of Plant Breeding and Acclimatization Institute-NRI of WOSR form a distinctly different group on the dendrogram (Fig. 3) from other double low WOSR. The genetic diversity of resynthesized (RS) and semi-resynthesized (semi-RS) lines has also been demonstrated in studies of 101 breeding 
lines and WOSR cultivars using 10 AFLP primer combinations (Liersch et al. 2016). Similar results were obtained by Sosnowska et al. (2017) in investigations of collections of spring and WOSR cultivars, B. rapa and Brassica oleracea, as well as nearly $50 \mathrm{RS}$ lines and semi-RS lines. Confirmation of the distinctiveness of resynthesized lines by AFLP markers shows that these lines can be valuable material for creating separate gene pools, especially for hybrid breeding of WOSR (Szała et al. 2016; Sosnowska et al. 2017). Jesske et al. (2013) analyzed 126 genotypes including 55 natural B. napus genotypes (winter, spring, Asian), and vegetable/cabbage forms and 71 resynthetic lines using 20 AFLP primer combinations. The authors showed a high value of GD between these groups, and thus created a dendrogram of rapeseed groups to clusters according to their origin and type/form in contrast to the resynthetic lines (RS) that created completely separate clusters. Seyis et al. (2006), Girke et al. (2012a, b), Jesske et al. (2013), and Chatterjee et al. (2016) in their research confirmed the usefulness of new RS rapeseed genotypes created as a result of hybridizing $B$. rapa with $B$. oleracea diploid parents. Hybridization helped to broaden the variability of breeding material, and to create a gene pool for use by breeders (Tonosaki et al. 2016). These authors point to the high yield potential of hybrids created with rapeseed RS lines, as confirmed by results from field experiments conducted in several environments.

The presented results confirmed the usefulness of molecular markers from the AFLP technique for the study of the GD of various WOSR collections. This resulted from the large number of generated markers and the repeatability of the obtained results. In the case of the first collection, 11 combinations of AFLP primers were used for the study, and in the second collection seven combinations allowed the characterization of the rapeseed genotypes tested and the creation of separate heterotic groups. Determination of genetic variation based on molecular markers is particularly useful in breeding F1 hybrid cultivars, an example of which is a winter rapeseed restored hybrid-Poznaniak-developed on the basis of the CMS ogura system, whose parental components (MS $83 \times$ BR 26) were selected from different clusters (data not published). Understanding the genetic variability of breeding materials is a valuable complement to classical breeding methods. It allows the selection of the best components for creating test hybrids, often eliminating, at the early stages of breeding, genotypes that are unsuitable from the point of view of breeding. This approach enables the comprehensive selection of CMS ogura lines and restorer lines which will be used for further crosses and then evaluated for general and specific combination ability in multi-experimental field trials (Nowosad et al. 2016, 2017; Bocianowski et al. 2018, 2019). In addition, demonstrating the significant genetic separateness of the resynthesized lines (RS) of rapeseed from commonly cultivated double low WOSR cultivars will enable a broadening of the variability within Brassica and the use of RS lines in breeding new winter rapeseed cultivars (Szała et al. 2016; Sosnowska et al. 2017).

Acknowledgements The authors thank Plant Breeding Company Strzelce Ltd. for plant materials used in this study. We thank also Ph.D., M. Dabert and her team for technical support (Molecular Biology Techniques Laboratory, Faculty of Biology, Adam Mickiewicz University). Financial support for this work was provided by the Ministry of Agriculture and Rural Development, Poland "Biological Progress in Plant Breeding" (2007-2013).

\section{Compliance with ethical standards}

Conflict of interest The authors declare that they have no conflict of interest.

Open Access This article is distributed under the terms of the Creative Commons Attribution 4.0 International License (http:// creativecommons.org/licenses/by/4.0/), which permits unrestricted use, distribution, and reproduction in any medium, provided you give appropriate credit to the original author(s) and the source, provide a link to the Creative Commons license, and indicate if changes were made.

\section{References}

Ali M, Copeland LO, Elias SG, Kelly JD (1995) Relationship between genetic distance and heterosis for yield and morphological traits in winter canola (Brassica napus L.). Theor Appl Genet 91:118-121

Bocianowski J, Nowosad K, Bujak H, Łuczkiewicz T, Piesik D (2016) Evaluation of the breeding value of the spring oilseed rape (Brassica napus L.) inbred lines based on a multitrait analysis. Indian J Genet Pl Br 76:284-289

Bocianowski J, Nowosad K, Liersch A, Popławska W, Łącka A (2018) Genotype-by-environment interaction for seed glucosinolate content in winter oilseed rape (Brassica napus L.) using an additive main effects and multiplicative interaction model. Biom Lett 55:85-96

Bocianowski J, Niemann J, Nowosad K (2019) Genotype-byenvironment interaction for seed quality traits in 
interspecific cross-derived Brassica lines using additive main effects and multiplicative interaction model. Euphytica 215:7

Chatterjee D, Banga S, Gupta M, Bharti Salisbury PA, Banga SS (2016) Resynthesis of Brassica napus trough hybridization between $B$. juncea and B. carinata. Theor Appl Genet 129:977-990

Chèvre AM, Delourme R, Eber F, Arus P (1991) First results of rapeseed variety identifications by isozyme electrophoresis. Cruciferae Newslett 14-15:70-71

Delourme R, Falentin C, Fomeju BF, Boillot M, Lasalle G, André I, Duarte J, Gauthier V, Lucantc N, Marty A, Pauchon M, Pichon JP, Ribière N, Trotoux G, Blanchard P, Rivière N, Martinant JP, Pauquet J (2013) High-density SNP-based genetic map development and linkage disequilibrium assessment in Brassica napus L. BMC Genom 14:120

Doyle JJ, Doyle JL (1990) Isolation of plant DNA from fresh tissue. Focus 12:13-15

Ecke W, Clemens R, Honsdorf N, Becker HC (2010) Extent and structure of linkage disequilibrium in canola quality winter rapeseed (Brassica napus L.). Theor Appl Genet 120:921-931

Excoffier L, Smouse P, Quattro J (1992) Analysis of molecular variance inferred from metric distances among DNA haplotypes: application to human mitochondrial DNA restriction data. Genetics 131:479-491

Faltusová Z, Kučera L, Ovesná J (2011) Genetic diversity of Brassica oleracea var. capitate gene bank accessions assessed by AFLP. Electron J Biotechnol 14(3). https://doi. org/10.2225/vol.14-issue3-fulltext-4, http://www.ejbiotec hnology.info/index.php/ejbiotechnology/article/viewFile? v14n3-4/1301. Accessed 8 Mar. 2016

Fu D, Xiao M, Hayward A, Fu Y, Liu G, Jiang G, Zhang H (2014) Utilization of crop heterosis: a review. Euphytica 197:161-173

Gao C, Yin J, Mason AS, Tang Z, Ren X, Li C, An Z, Fu D, Li J (2014) Regularities in simple repeat variations induced by a cross of resynthesized Brassica napus and natural Brassica napus. Plant Omics J 7:35-46

Girke A, Schierholt A, Becker HC (2012a) Extending the rapeseed genepool with resynthesized Brassica napus L. I: genetic diversity. Genet Resour Crop Evol 59:1441-1447

Girke A, Schierholt A, Becker HC (2012b) Extending the rapeseed genepool with resynthesized Brassica napus L. II: heterosis. Theor Appl Genet 124:1017-1026

Irzykowska L, Bocianowski J (2008) Genetic variation, pathogenicity and mycelial growth rate differentiation between Gaeumannomyces graminis var. tritici isolates derived from winter and spring wheat. Ann Appl Biol 152:369-375

Jesske T, Olberg B, Schierholt A, Becker HC (2013) Resynthesized lines from domesticated and wild Brassica taxa and their hybrids with B. napus L.: genetic diversity and hybrid yield. Theor Appl Genet 126:1053-1065

Krystkowiak K, Adamski T, Surma M, Kaczmarek Z (2009) Relationship between phenotypic and genetic diversity of parental genotypes and the specific combining ability and heterosis effects in wheat (Triticum aestivum L.). Euphytica 165:419-434
Krzymińska A, Gawłowska M, Wolko B, Bocianowski J (2008) Genetic diversity of ornamental Allium species and cultivars assessed with isozymes. J Appl Genet 49:213-220

Lefort-Buson M, Hebert Y, Damerval C (1988) Les outils d'évaluation de la divérsité génétique et phénotypique. Agronomie 8:173-178

Li L, Wanapu C, Huang X, Huang T, Li Q, Peng Y, Huang G (2011) Comparison of AFLP and SSR for genetic diversity analysis of Brassica napus hybrids. J Agric Sci 3:101-110

Liersch A, Bocianowski J, Kozak M, Bartkowiak-Broda I (2013) Comparison of isozyme, RAPD and AFLP markers in genetic similarity assessment of CMS ogura $\mathrm{F}_{1}$ hybrids of winter oilseed rape (Brassica napus L.) parental lines. Acta Biol Cracov Bot 55:49-57

Liersch A, Bocianowski J, Woś H, Szała L, Sosnowska K, Cegielska-Taras $\mathrm{T}$, Nowosad $\mathrm{K}$, Bartkowiak-Broda I (2016) Assessment of genetic relationship on breeding lines and cultivars of Brassica napus and their implications for breeding winter oilseed rape. Crop Sci 56:1540-1549

Lombard V, Delourme R (2001) A consensus linkage map for rapeseed (Brassica napus L.): construction and integration of three individual maps from DH populations. Theor Appl Genet 103:491-507

Luo X, Ma C, Yi B, Tu J, Shen J, Fu T (2016) Genetic distance revealed by genomic single nucleotide polymorphism and their relationships with harvest index heterotic traits in rapeseed (Brassica napus L.). Euphytica 209:41-47

Mikołajczyk K (2013) Genetic markers in oilseed rape breeding programs. PBAI-NRI, monographs and dissertations No. 40:7-119

Nei M, Li W (1979) Mathematical model for studying genetic variation in terms of restriction endonucleases. Proc Natl Acad Sci USA 76:5269-5273

Nowosad K, Liersch A, Popławska W, Bocianowski J (2016) Genotype by environment interaction for seed yield in rapeseed (Brassica napus L.) using additive main effects and multiplicative interaction model. Euphytica 208:187-194

Nowosad K, Liersch A, Poplawska W, Bocianowski J (2017) Genotype by environment interaction for oil content in winter oilseed rape (Brassica napus L.) using additive main effects and multiplicative interaction model. Indian J Genet Pl Br 77:293-297

Nurhasanah, Ecke W (2016) Construction of a high fidelity genetic linkage map using AFLP and SSR markers in rapeseed (Brassica napus L.). SABRAO J Breed Genet 48:189-199

Peakall R, Smouse PE (2012) GenAIEx 6.5: genetic analysis in excel. Population genetic software for teaching and research-an update. Bioinformatics 28:2537-2539

Rahman H, Bennett RA, Séguin-Swartz G (2015) Broadening genetic diversity in Brassica napus canola: development of canola-quality spring $B$. napus from $B$. napus x B. oleracea var. alboglabra interspecific crosses. Can J Plant Sci 95:29-41

Rakoczy-Trojanowska M, Krajewski P, Bocianowski J, Schollenberger M, Wakuliński W, Milczarski P, Masojć P, Targońska-Karasek M, Banaszak Z, Banaszak K, Brukwiński W, Orczyk W, Kilian A (2017a) Identification of single nucleotide polymorphisms associated with brown rust resistance, $\boldsymbol{\alpha}$-amylase activity and pre-harvest 
sprouting in rye (Secale cereale L.). Plant Mol Biol Rep 35:366-378

Rakoczy-Trojanowska M, Orczyk W, Krajewski P, Bocianowski J, Stochmal A, Kowalczyk M (2017b) $S c B x$ gene based association analysis of hydroxamate content in rye (Secale cereale L.). J Appl Genet 58:1-9

Reif JC, Melchinger AE, Xia XC, Warburton ML, Hoisington DA, Vasal SK, Beck D, Bohn M, Frish M (2003) Use of SSRs for establishing heterotic groups in subtropical maize. Theor Appl Genet 107:947-957

Seidler-Łożykowska K, Kuczyńska A, Mikołajczyk K, Nowakowska J, Bocianowski J (2014) Estimation of genetic distance among genotypes of caraway (Carum carvi L.) using RAPD-PCR. Acta Sci-Agron 36:183-188

Seyis F, Friedt W, Lühs W (2006) Yield of Brassica napus L. hybrids developed using resynthesized rapeseed material sown at different locations. Field Crop Res 96:176-180

Snowdon RJ, Friedt W (2004) Molecular markers in Brassica oilseed breeding: current status and future possibilities. Plant Breeding 123:1-8

Sosnowska K, Cegielska-Taras T, Liersch A, Karłowski WM, Bocianowski J, Szała L, Mikołajczyk K, Popławska W (2017) Genetic relationships among resynthesized, semiresynthesized and natural Brassica napus L. Euphytica 213:212

Stępień Ł, Mohler V, Bocianowski J, Koczyk G (2007) Assessing genetics diversity of Polish wheat (Triticum aestivum) varieties using microsatellite markers. Genet Resour Crop Ev 54:1499-1506

Szała L, Sosnowska K, Popławska W, Liersch A, Olejnik A, Kozłowska K, Bocianowski J, Cegielska-Taras T (2016) Development of new restorer lines for CMS ogura system with the use of resynthesized oilseed rape (Brassica napus L.). Breeding Sci 66:516-521
Tonosaki K, Osabe K, Kawanabe T, Fujimoto R (2016) The importance of reproductive barriers and the effect of allopolyploidization on crop breeding. Breeding Sci 66:333-339

Varghese G, Jose M, Dinesh Raj R, Bocianowski J, Thomas G, Omanakumari N (2014) Quantitative and molecular analyses reveal a deep genetic divergence between the ancient medicinal rice (Oryza sativa) Njavara and syntopic traditional cultivars. Ann Appl Biol 164:95-106

Vos P, Hogers R, Bleeker M, Reijans M, van de Lee T, Hornes M, Frijters A, Pot J, Teleman J, Kuiper M, Zabeau M (1995) AFLP: a new technique for DNA fingerprinting. Nucl Acids Res 23:4407-4414

Wittkop B, Snowdon RJ, Friedt W (2009) Status and perspectives of breeding for enhanced yield and quality of oilseed crops for Europe. Euphytica 170:131-140

Wolko Ł, Bocianowski J, Antkowiak W, Słomski R (2015) Genetic diversity and population structure of wild pear [Pyrus pyraster (L) Burgsd.] in Poland. Open Life Sci 10:19-29

Wu J, Li F, Xu K, Gao G, Chen B, Yan G, Wang N, Qiao J, Li J, Li H, Zhang T, Song W, Wu X (2014) Assessing and broadening genetic diversity of a rapeseed germplasm collection. Breeding Sci 64:321-330

Yu CY, Hu SW, Zhao HX, Guo AG, Sun GL (2005) Genetic distance revealed by morphological characters, isozymes, proteins and RAPD markers and their relationships with hybrid performance in oilseed rape (Brassica napus L.). Theor Appl Genet 110:511-518

Publisher's Note Springer Nature remains neutral with regard to jurisdictional claims in published maps and institutional affiliations. 